\title{
Synthetic Lethal Screen to Identify Molecular Mechanisms that Drive Uveal Melanoma
}

\author{
Kristin Lee Recker ${ }^{1}$, Sharon Norton ${ }^{1}$, Amin Sobh ${ }^{1}$, Alberto Riva ${ }^{1}$, Kenneth \\ Chang $^{2}$, Jeffim Kuznetsov ${ }^{3}$, Michael Durante ${ }^{3}$, Fernanda Flores ${ }^{4}$, Richard L. \\ Bennett $^{1}$, Christopher Vakoc ${ }^{2}$, Kieran Smalley ${ }^{4}$, William Harbour ${ }^{3}$, and \\ Jonathan D. Licht ${ }^{1}$ \\ ${ }^{1}$ University of Florida, ${ }^{2}$ Cold Spring Harbor Laboratory, ${ }^{3}$ University of Miami, ${ }^{4}$ Moffitt Cancer Center
}

Faculty mentor: Richard L. Bennett, Department of Medicine, Division of Hematology \& Oncology

\begin{abstract}
Uveal melanoma is a highly aggressive form of cancer that exhibits a propensity to metastasize to the liver and represents about $5 \%$ of all melanoma cases. At this time there are no effective treatments for metastatic uveal melanoma and new therapeutic strategies are urgently needed. The majority of uveal melanomas are derived from early activating mutations in the G-proteins GNA11 or GNAQ. To identify molecular mechanisms that uveal melanoma cell lines rely upon for proliferation and survival, we performed CRISPR/Cas9 mediated synthetic lethality screening. To accomplish this, we generated a uveal melanoma cell line, 92.1, that expressed the endonuclease Cas9 and introduced a guide RNA library into these cells that targets the functional domains of epigenetic regulator proteins. Thus, epigenetic regulator genes that were required for the survival and proliferation of these cells were determined by measuring which guide RNAs "dropped out" over time. Our results revealed that ATAT1, BRD4, CHD4, DOT1L, HDAC1, and TIP60 were strongly selected for in the 92.1 uveal melanoma cell line. By examination of the STRING biological database of known and predicted protein-protein interactions, we found that almost all of the genes found essential in the uveal melanoma cell line 92.1 interact with HDAC1 and DOT1L. Thus, future experiments may now include testing the efficacy of clinically available DOT1L and/or HDAC inhibitors in the context of uveal melanoma.

Keywords: CRISPR/Cas9, uveal melanoma, epigenetic sgRNA library, synthetic lethal screen
\end{abstract}

\section{Introduction}

Although rare, uveal melanoma (UM) currently represents the most common primary intraocular cancer. It arises from melanocytes within the eye that can and often will metastasize to the liver (Damato \& Coupland, 2012). Because the exact cause is unknown, there are currently no effective therapeutic strategies for the treatment of this disease. However, research has shown that gain-of-function mutations in the alpha subunits of trimeric G-proteins like GNA11 and GNAQ play a crucial role in the majority of uveal melanoma cases (Van Raamsdonk et al., 2010). A study by Feng et al. has previously shown that the abnormal activity of G proteins is in 
part due to the activation of YAP, a transcriptional coactivator in the Hippo pathway, which has been shown to play an important role in the development of several cancers (Feng et al., 2019; Steinhardt et al., 2008; Xu et al., 2009; Zhao, Li, \& Guan, 2010; Zhao et al., 2007). Further investigation is needed in order to determine the epigenetic consequences and precise molecular mechanisms involved in these activating mutations.

With huge technological advances in gene editing tools like the CRISPR-Cas9 system, the ability to determine precise genes that cells depend on for growth and proliferation has been greatly enhanced (Wang et al., 2015). The powerful CRISPR-Cas9 screening of protein domains in particular has allowed several lethality screens to be performed and analyzed on genomicwide scales so that we may be able to determine specific cancer gene dependencies. While there are several approaches to performing synthetic lethality screens, they serve a common goal of identifying gene function across various cell lines. In our CRISPR-Cas9 knockout approach, we transduced the uveal melanoma cell line 92.1 with a pool of single-guide RNA vectors that targeted thousands of genes coding for specific proteins. After allowing time for the depletion of sgRNAs that targeted essential genes, we isolated the genomic DNA and used massive parallel sequencing to determine which of those genes were required for the growth of this UM cell line. The degree to which certain regions or genes within the genome were depleted could help to establish the functional importance of those individual domains and introduce us to new targeted therapeutic drug strategies (Shi et al., 2015). Programs like DepMap, the Cancer Dependency Map, have been designed to characterize which genes are commonly essential across several cancer cell lines and which ones are strongly selected for within certain cancers (O'Neil, Bailey, $\&$ Hieter, 2017; Tsherniak et al., 2017). By determining which genes are necessary for the growth of different types of cancers, we may be better equipped to understand their function and, more importantly, how we can use drugs to target them as potential anti-cancer therapies. The collection of this data is crucial to the understanding of gene function, the effects of drug therapies, and the mechanisms that drive several cancers.

To identify essential genes in uveal melanoma cells and new genetic vulnerabilities, we stably expressed the Cas9 endonuclease in the uveal melanoma cancer cell line 92.1 that harbors mutant GNAQ. These cells were subsequently transduced with a guide RNA library developed by Dr. Chris Vakoc (Cold Spring Harbor Laboratory, NY) that targets the functional domains of epigenetic regulator genes (1390 sgRNAs against 192 genes). The sgRNAs that significantly 
decreased over time were measured to determine which genes were essential for the proliferation and growth of the uveal melanoma cell line (Shi et al., 2015). We chose to use epigenetic regulatory proteins as our targets because the role of epigenetics in the growth and development of uveal melanoma remains relatively unclear, and drugs targeting these domains have yet to be used in the clinic for $\mathrm{UM}(\mathrm{Li}, \mathrm{Jia}, \& \mathrm{Ge}, 2017)$. This synthetic lethality approach allowed us for the first time to investigate how activating GNAQ mutations may utilize the epigenetic landscape to drive aberrant growth, and results may be used to guide future therapeutic decision making.

\section{MATERIALS AND METHODS}

\section{Cell Lines}

The GNAQ mutant uveal melanoma cell line 92.1 was obtained from Dr. William Harbour at the University of Miami Bascom Palmer Eye Institute. These cells were grown in 100mm tissue culture disks in Advanced RPMI with $10 \%$ FBS, $1 \%$ Pen/strep, $1 \%$ Glutamax at $37^{\circ} \mathrm{C}$ in a $5 \% \mathrm{CO}_{2}$ incubator, and Lentivirus was used to transduce these cells with Cas9 and blasticidin resistance. Cells containing Cas9 were maintained in $10 \mu \mathrm{g} / \mathrm{ml}$ of blasticidin.

\section{Protein Expression}

SDS-PAGE with a 4-12\% gradient stacking gel was performed on cell lysates to measure Cas9 protein expression. The antibody against Cas9 (Cat\#14697S from Cell Signaling Technologies) was used at 1:1000 dilution and antibody to GAPDH (Cat\#sc-25778 from Santa Cruz Biotechnologies) was used at 1:1000 dilution as a loading control. Immunoblots were visualized on a Li-Cor Odyssey.

\section{Measurement of Cas9 Efficiency}

All 92.1 cells that expressed Cas 9 were transduced with lentiviral vector expressing both green fluorescence protein (GFP) and guide RNAs targeting either PCNA, RPA1, or the ROSA26 locus in a 6-well plate. Flow cytometry using an Accuri C6 flow cytometer was performed to measure the percentage of cells GFP+ at 2, 6, 9, and 12 days after transduction with the GFP-gRNA virus vector.

\section{Screening with the epigenetic sgRNA library}

The sgRNA library DNA was transfected into 293T cells to package into lentivirus. Using a six-well plate, we transduced six populations of cells with $0,2,5,10,25 \mu \mathrm{L}$ of the 10 -fold diluted virus. After 48 hours, the percentage of cells that were GFP+ was measured using flow cytometry. After determining the proper titer of lentivirus to ensure a MOI of $0.3,150 \mathrm{~mm}$ plates 
of 92.1 cells was transduced at $1000 \mathrm{X}$ coverage of a sgRNA library directed against the functional domains of epigenetic regulators. Cells were collected two days after transduction and after nine cell doublings (14 days after transduction).

\section{Sequencing and Analysis}

The sgRNA libraries were amplified by PCR and sequencing libraries prepared by adding variable length barcode adapters. The libraries were sequenced by Novogene. The sgRNAs were sequenced with a depth of $>20000 X$. By calculating the number of reads that aligned to each sgRNA, the Model-based Analysis of Genome-wide CRISPR/Cas9 Knockout (MAGeCK) program was used to determine which sgRNAs were severely depleted.

\section{RESULTS}

\section{Development of a uveal melanoma cell line that expressed active Cas9 endonuclease}

In order to investigate specific epigenetic gene dependencies in cells with the activating Gprotein mutations implicated in a majority of uveal melanoma cancers, a CRISPR/Cas9-mediated synthetic lethal screen was used (Figure 1). First, the 92.1 uveal melanoma cell line was transduced with lentivirus vector containing Cas9 and a blasticidin resistance gene. Expression of Cas9 was confirmed by a Western blot on extracts of cells that had been selected in blasticidin for one week (Figure 2).

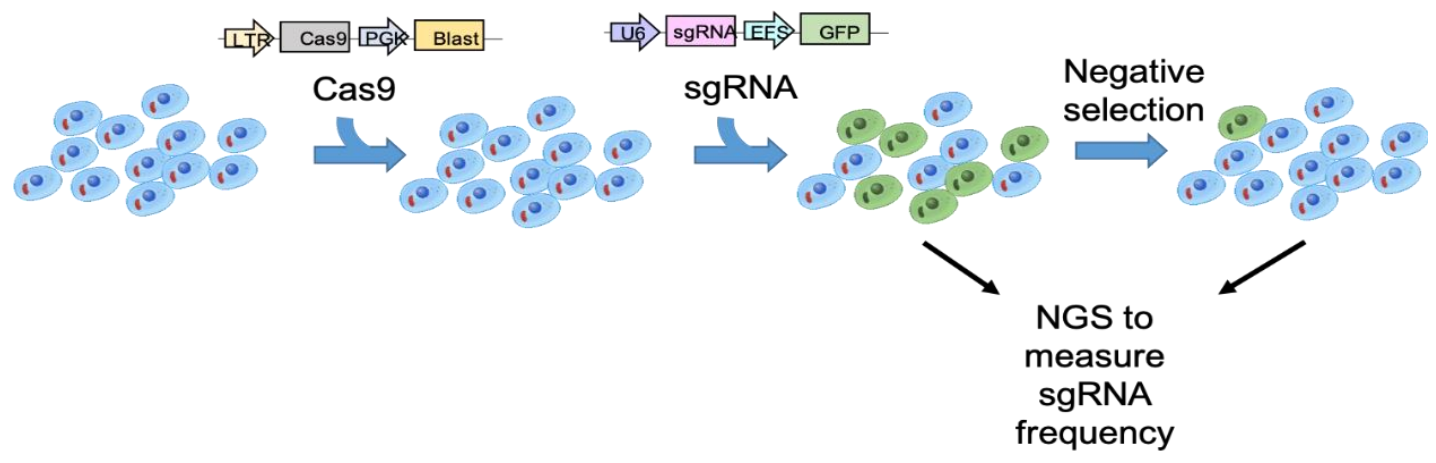

Figure 1. The uveal melanoma cell line 92.1 was first transduced with lentivirus containing the Cas 9 vector. After ensuring that to light. Cas9 mediated cleavage of essential genes targeted by the sgRNA allowed for negative Cas9 was stably expressed by selecting cells in blasticidin, the cells were transduced with a vector containing a specific guide RNA and green fluorescence protein (GFP) that exhibits bright green fluorescence when exposed selection. Since GFP and sgRNA abundance were linked, we can monitor drop out of cells with a sgRNA to an essential gene by observing the decrease of GFP over time. Finally, after the screen was complete with a sgRNA library, next-generation sequencing (NGS) allowed us to determine which sgRNAs changed over time and which genes were essential for growth. 


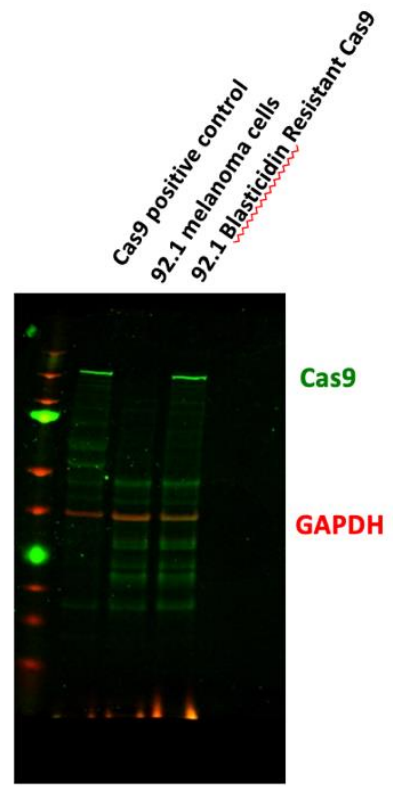

Figure 2. A Western Blot membrane treated with Cas9 and GAPDH antibodies prior to being treated with secondary fluorescent antibodies (green and red, respectively) was performed. GAPDH acted as a loading control for all cell lines included. We compared the 92.1 uveal melanoma cell line transduced with blasticidin resistance to a sample of untreated 92.1 melanoma cells. This was done to ensure that Cas9 was stably expressed.

The picture to the left indicates that the transduction was successful.

To determine whether the Cas9 had sufficient endonuclease efficiency to perform our screen with a sgRNA library, three control single guide RNAs (sgRNAs) targeting either PCNA, RPA1, or the ROSA26 locus were tested. Protein PCNA, a DNA clamp, and RPA1, a single-stranded binding protein, are both proteins involved in DNA replication and are therefore essential for the continuing function of the cell. However, disruption of the ROSA26 locus, which is involved in the ubiquitous expression of transgenes, should not affect the cell's ability to grow (Chen, Krohn, Bhattacharya, \& Davies, 2011). The effects of these three control sgRNAs were monitored over a twelve-day period by recording the percentage of cells that were GFP+ and therefore carried the plasmid expressing the sgRNA at day 2, 6, 9, and 12 using flow cytometry. As expected, both the PCNA and RPA1-targeted sgRNAs were lethal to the cells and dropped out, while cleavage of the sgRNAs targeting the ROSA26 locus did not cause cell death and persisted in cells (Figure 3). 


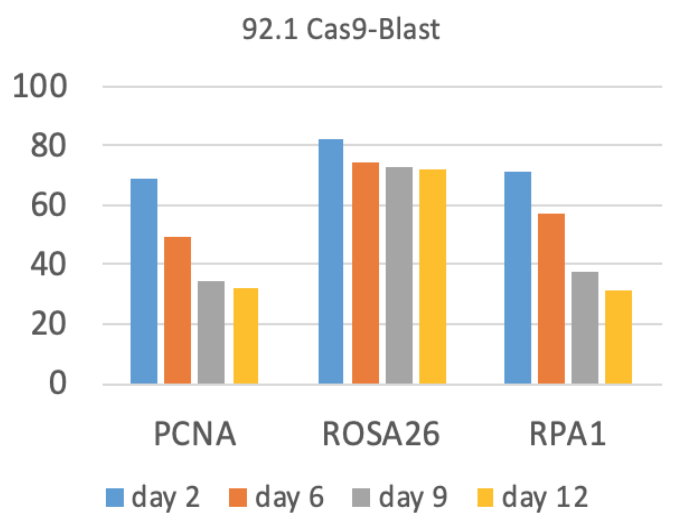

Figure 3. To show that the Cas 9 endonuclease was properly working, control sgRNAs were each introduced into the cell line. Cas9 mediated cleavage of the sgRNAs targeting ROSA26 should not be lethal to the cell, while those of the sgRNAs targeting PCNA and RPA1 should cause cell death. This is because PCNA and RPA1 are essential proteins used in DNA replication. The bar graphs above exhibit a significant decrease of the PCNA and RPA1 sgRNAs over time when compared to ROSA26. This in turn exhibits that Cas9 is working.

\section{Optimization of CRISPR-Cas9 screening conditions in 92.1 cells}

To determine an appropriate time period over which to conduct the lethality screen, we measured the time it took for the 92.1 cells to double. Previous reports suggest that for best results synthetic lethality screens should be performed for approximately 10 cell doublings. The 92.1 cells took approximately 38.96 hours, or 1 1/2 days, to double (Figure 4). In order to ensure that the sgRNAs were fully effective and that essential genes were being knocked out, the screen was conducted over 14 days to allow the cells to double approximately nine times.

Next, a titration of the epigenetic single-guide RNA library was performed using various amounts of the lentivirus to determine the concentration of virus to use for the synthetic lethal screen. Forty-eight hours after the transduction of cells with incremental increases in the amount of virus, the percentage of cells that were GFP+ was measured using flow cytometry (Figure 5). Thus, roughly $5 \mu \mathrm{L}$ of lentivirus was determined to yield a multiplicity of infection (MOI) of 0.3 . Therefore, this amount was used for our lethality screen in order to limit the likelihood of any cell being transduced with more than one guide RNA. 


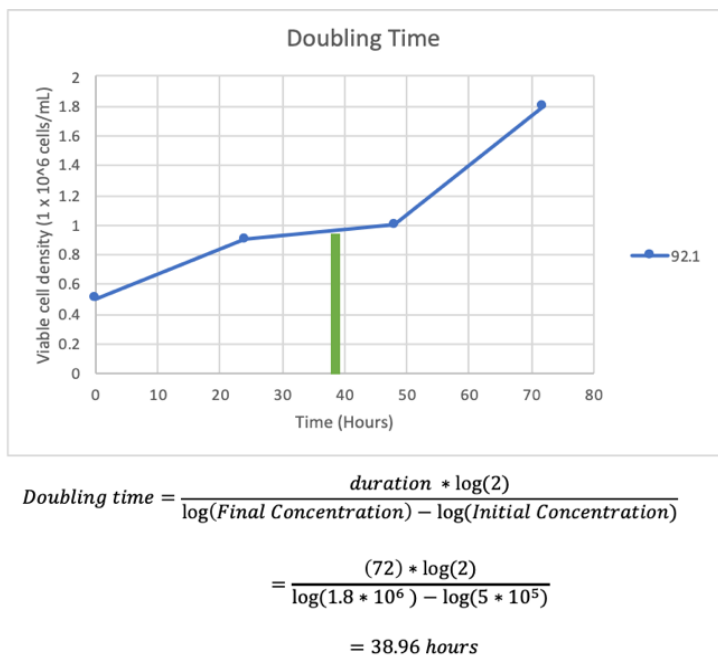

Figure 4. Doubling time of 92.1 melanoma cell line revealed that it takes approximately 38.96 hours for the population of 92.1 cells to double.

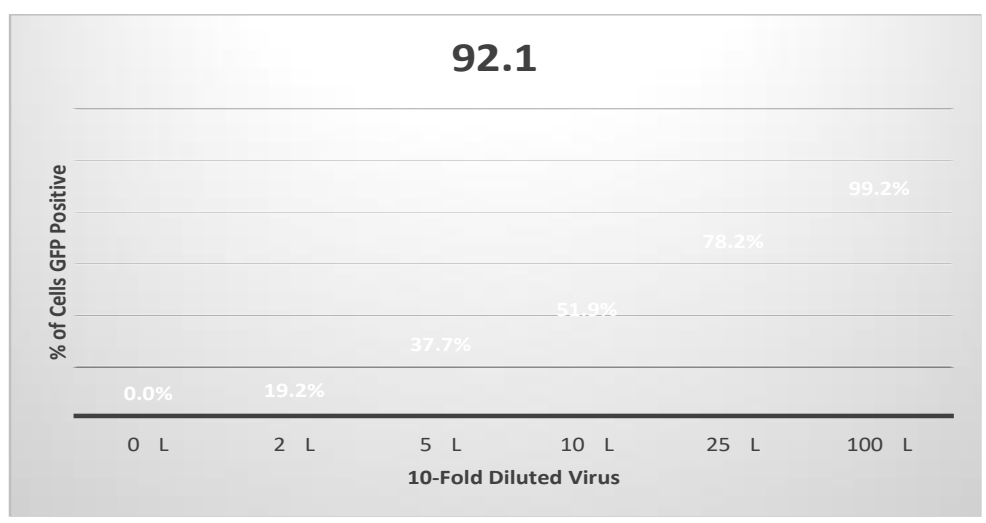

Figure 5. In order to ensure a 1000x coverage of cells (each sg RNA in at least 1000 cells), a titration was performed using different amounts of the guide RNA library vector. By determining the concentration where the ratio of the control sgRNAs to infection targets was .33 , it was ensured that each cell would be infected by only one sgRNA.

\section{Synthetic lethal screen of 92.1 cells reveals that HDAC1 and DOT1L are essential genes}

Synthetic lethal screening of 92.1 cells was performed at 1000X coverage using a sgRNA library directed against the functional domains of epigenetic regulators (Shi, J. et al., 2015). Cells were collected two days after transduction and after nine cell doublings (14 days after transduction). These two samples of cells were then sequenced using massively parallel nextgeneration sequencing as shown in Figure 6. The sgRNAs were amplified and sequenced with a depth of $>20000 X$. The screen was repeated one time to yield two biological replicates. 


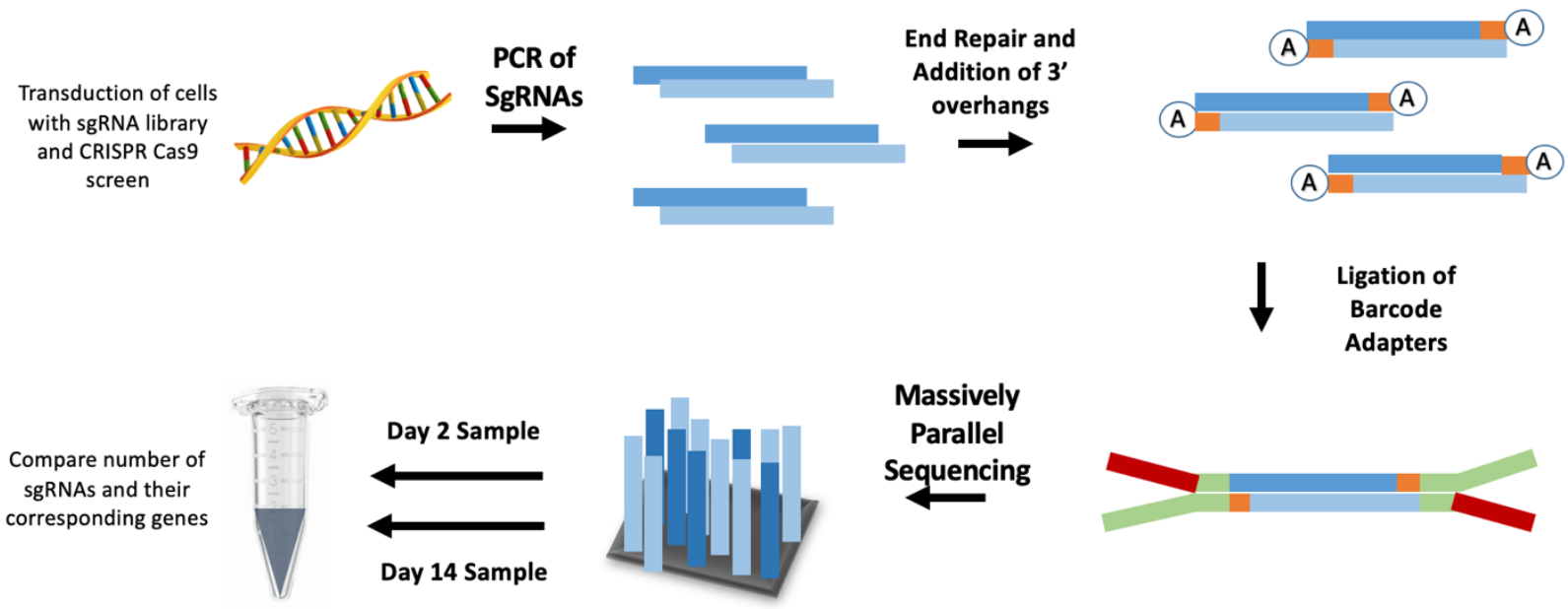

Figure 6. After transducing the cells and performing the synthetic lethality screen, the sgRNAs of the 92.1 cells were amplified and sequenced with a depth of $>20000 \mathrm{X}$. They were collected at 2 days after transduction and after 9 cell doublings (14 days after transduction). The number of sgRNAs and their targeted genes were compared across both samples.

\begin{tabular}{lcc}
\multicolumn{3}{l}{ Table 1. Combined Score from Both Replicates } \\
\hline Gene & $\beta$ & $\begin{array}{c}\text { False } \\
\text { Discovery } \\
\text { Rate (FDR) }\end{array}$ \\
\hline PRL9 & -0.86228 & 0 \\
TIP60 & -0.39917 & 0 \\
BRD4 & -0.33019 & 0 \\
SETD1A & -0.26015 & 0 \\
BRD8 & -0.24682 & 0 \\
JMJD8 & -0.23016 & 0.090909 \\
SETD1B & -0.2301 & 0.090909 \\
TRIM28 & -0.20566 & 0.090909 \\
RPA3 & -0.34223 & 0.14286 \\
SCRAP & -0.19771 & 0.14286 \\
ATAT1 & -0.18622 & 0.22222 \\
BRD2 & -0.1485 & 0.22222 \\
POLR2D & -0.3568 & 0.22727 \\
RPL23A & -0.31053 & 0.22727 \\
POLR2A & -0.20555 & 0.22727 \\
CHD4 & -0.16412 & 0.23077 \\
SMARCA4 & -0.16197 & 0.23077 \\
\hline
\end{tabular}

The Model-based Analysis of Genome-wide CRISPR/Cas9 Knockout (MAGeCK) program was used to compare the number of reads that aligned to each sgRNAs of each gene at day 14 versus day 2. This gene ranking tool helps to prioritize which genes are most important for proliferation and growth based on beta scores and false discovery rates (FDR), as shown in Table 
1. The beta scores reflect the strength and magnitude of depletion between the initial and final timepoints. Thus, the more negative the beta score, the more important it is for the viability of the 92.1 cancer cell line. In addition, the FDR represents the p-value adjusted for multiple comparisons which tells us how significant each beta score truly is ( $\mathrm{Li}$ et al., 2014). Using the combined scores of both replicates, 18 essential genes were identified in the 92.1 cancer cell line (Table 2).

\begin{tabular}{|c|c|c|c|c|}
\hline \multirow[t]{2}{*}{$\underline{\text { Gene }}$} & \multicolumn{4}{|c|}{ Number of sgRNAs } \\
\hline & Day 2 & Day 14 & Day 2 - Day 14 & $\begin{array}{l}\text { Percent } \\
\text { Depleted }\end{array}$ \\
\hline$B R D 4$ & 41,694 & 11,909 & 29,785 & $71.44 \%$ \\
\hline$H D A C 1$ & 14,939 & 6,602 & 8,337 & $55.81 \%$ \\
\hline$J M J D 8$ & 32,530 & 25,674 & 6,856 & $21.08 \%$ \\
\hline$K M T 2 D$ & 22,849 & 13,849 & 9,000 & $39.39 \%$ \\
\hline SMARCA4 & 17,040 & 8,443 & 8,597 & $50.45 \%$ \\
\hline TRIM28 & 18,667 & 14,805 & 3,862 & $20.69 \%$ \\
\hline SETDIB & 44,528 & 16,399 & 28,129 & $63.17 \%$ \\
\hline$B R D 2$ & 46,463 & 17,002 & 29,461 & $63.41 \%$ \\
\hline TIP60 & 31,161 & 7,385 & 23,776 & $76.30 \%$ \\
\hline$B R D 8$ & 36,018 & 9,176 & 26,842 & $74.52 \%$ \\
\hline SUV420H1 & 40,548 & 17,565 & 22,983 & $56.68 \%$ \\
\hline SETDIA & 34,489 & 16,790 & 17,699 & $51.32 \%$ \\
\hline DOTIL & 43,835 & 23,131 & 20,704 & $47.23 \%$ \\
\hline SETD8 & 16,849 & 6,336 & 10,513 & $62.40 \%$ \\
\hline CHD4 & 39,567 & 15,912 & 23,655 & $59.78 \%$ \\
\hline$M O Z$ & 37,756 & 16,162 & 21,594 & $57.19 \%$ \\
\hline ATAT1 & 52,232 & 13,835 & 38,397 & $73.51 \%$ \\
\hline EHMT1 & 33,948 & 17,574 & 16,374 & $48.23 \%$ \\
\hline
\end{tabular}

To determine if the 18 genes that were found to be essential in 92.1 cells were specifically essential to this uveal melanoma cell line or universally essential in all cell lines, the Cancer Dependency Map, a collection of CRIPSR-Cas9 essentiality screens from over 500 different cancer cell lines that has been sorted and analyzed using a precise calculation, was used (Broad Institute, n.d.). In Table 3, a comparison was made between essential genes (highlighted in green) and those that were strongly selected for in our lethality screen. Four of the 18 genes were found to be commonly essential in at least $90 \%$ of cell lines, and therefore may be essential for growth across almost all cancers. The remaining 14 genes represent those that were more 
specifically essential in 92.1 cells and one gene, HDAC1, was strongly selected for in the uveal melanoma cell line. Thus, the dependency upon HDAC1 is 100 times more likely to have been sampled from a skewed distribution than a normal distribution indicating that it is unique to uveal melanoma.

\begin{tabular}{|c|c|c|c|}
\hline Gene & Description & $\begin{array}{l}\text { Dependent } \\
\text { cell lines }\end{array}$ & \\
\hline ATAT1 & Alpha Tubulin Acetyltransferase 1 & $1 / 517$ & \\
\hline BRD2 & Bromodomain Containing 2 & $229 / 517$ & \\
\hline BRD4 & Bromodomain Containing 4 & $499 / 517$ & $\begin{array}{l}\text { common } \\
\text { essential }\end{array}$ \\
\hline BRD8 & Bromodomain Containing 8 & $212 / 517$ & \\
\hline CHD4 & Chromodomain Helicase DNA Binding Protein 4 & $511 / 517$ & $\begin{array}{l}\text { common } \\
\text { essential }\end{array}$ \\
\hline DOT1L & DOT1 Like Histone Lysine Methyltransferase & $25 / 517$ & \\
\hline EHMT1 & Euchromatic Histone Lysine Methyltransferase 1 & $28 / 517$ & \\
\hline HDAC1 & Histone Deacetylase 1 & $15 / 517$ & $\begin{array}{l}\text { strongly } \\
\text { selective }\end{array}$ \\
\hline JMJD8 & Jumonji Domain Containing 8 & $0 / 517$ & \\
\hline KMT2D & Lysine Methyltransferase 2D & $202 / 517$ & \\
\hline $\mathrm{MOZ}$ & Lysine Acetyltransferase 6A (KAT6A) & $7 / 517$ & \\
\hline SCRAP & ???? & - & \\
\hline SETD1A & SET Domain Containing 1A & $498 / 517$ & $\begin{array}{l}\text { common } \\
\text { essential }\end{array}$ \\
\hline SETD1B & SET Domain Containing 1B & $67 / 517$ & \\
\hline SETD8 & Lysine Methyltransferase 5A & $98 / 517$ & \\
\hline SMARCA4 & $\begin{array}{c}\text { SWI/SNF Related, Matrix Associated, Actin } \\
\text { Dependent Regulator of Chromatin, Subfamily A, } \\
\text { Member } 4\end{array}$ & $180 / 517$ & \\
\hline SUV420H1 & Lysine Methyltransferase 5B (KMT5B) & $0 / 517$ & \\
\hline TIP60 & Lysine Acetyltransferase 5 (KAT5) & $481 / 517$ & $\begin{array}{l}\text { common } \\
\text { essential }\end{array}$ \\
\hline TRIM28 & Tripartite Motif Containing 28 & $54 / 517$ & \\
\hline
\end{tabular}

To determine how the top hits of our CRISPR-Cas9 screen may interact or be part of the same functional pathway the biological database of known and predicted protein-protein interactions, STRING, was used to generate a protein-protein interaction map. Of the novel genes that were strongly selected for in the 92.1 uveal melanoma cell line that included ATAT1, BRD4, CHD4, DOT1L, HDAC1, and TIP60. STRING analysis revealed that almost all of the genes found to be essential in the uveal melanoma cell line 92.1 interact with HDAC1 and DOT1L (Figure 7). 


\section{STRING Protein interaction map}

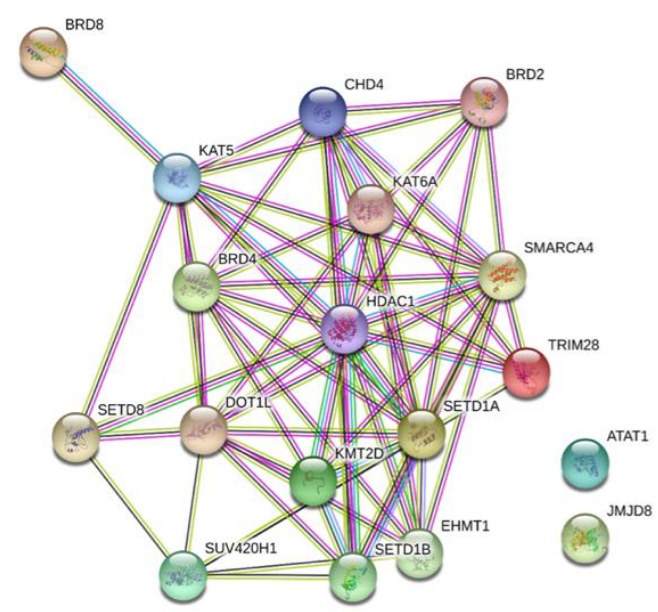

Figure 7. The biological database of known and predicted protein-protein interactions, STRING, was used to generate a protein-protein interaction map for proteins found to be significantly essential in at least one biological replicate of the sgRNA synthetic lethal screen in 92.1 cells. Almost all of the top hits from the essentiality screens tightly cluster around HDAC1 and DOT1L.

\section{Discussion}

Our lethality screen revealed that the genes ATAT1, BRD2, DOT1L, EHMT1, HDAC1, JMJD8, KMT2D, MOZ, SETDIB, SETD8, SMARCA4, SUV42OH and TRIM28 are essential in the 92.1 melanoma cell line but are not universally essential. Thus, these genes may be specifically important for growth and maintenance of uveal melanoma cell lines with GNAQ mutations. Almost all of the top hits from the essentiality screens tightly cluster around HDAC1 and DOT1L in protein-protein interaction analysis, and HDAC1 was considered our most selective hit by the cancer dependency map. Interestingly, DOT1L is a methyl transferase that catalyzes the methylation of histone H3 lysine 79, and inhibitors of DOT1L have been used therapeutically for multiple myeloma, acute leukemias, and more recently some breast cancers (Daigle et al., 2013; Ishiguro et al., 2019; Nassa et al., 2019; Stein et al., 2018). In addition, HDAC1 is a histone deacetylase, and inhibitors of HDAC1 have been used for multiple myeloma and T-cell lymphomas (Eckschlager, Plch, Stiborova, \& Hrabeta, 2017; Stubbs et al., 2015). Our results suggest that DOT1L and HDAC1 inhibitors may be therapeutically useful for uveal melanoma. Additional experiments must now be performed that test whether inhibitors targeting these genes are potential drug therapies for uveal melanoma.

The synthetic lethal screen performed here could be expanded by performing additional screens on other uveal melanoma cell lines that carry the GNAQ mutation. This would allow us to determine what epigenetic regulator genes are commonly essential across multiple uveal 
melanoma cell lines. In addition the screens performed in this experiment could be improved by screening for a longer time to allow more time for sgRNAs targeting essential genes to drop out and by accounting for copy number within the genome using the CERES score (Meyers et al., 2017). This calculation may help produce more accurate and precise results because sgRNAs that target genes with a high copy number may cause cell death due to the high degree of DNA damage produced after cutting the target gene rather than due to any function of the gene.

In the future, additional synthetic lethal screens will be performed with a sgRNA library targeting the entire genome. These screens can be performed in the presence or absence of various therapeutic agents such as MEK inhibitor to determine mechanisms of resistance and also to perhaps identify gene targets that would be useful for combination therapies. Together, these future experiments will allow us to identify new genetic vulnerabilities specific to uveal melanoma that may be targeted therapeutically.

\section{Acknowledgements}

I would like to especially thank Sharon Norton for teaching me lab techniques and performing the NGS library prep of the screen. In addition, I would like to thank my entire lab, including Darby Monagle, Gabe Prado, and Amin Sobh, for their patience and guidance on this journey; I would not have been able to do this without their help. Most importantly, I would like to thank Dr. Richard Bennett for his amazing mentorship and Dr. Jonathan Licht for the opportunity to work in his lab.

\section{References}

Daigle, S. R., Olhava, E. J., Therkelsen, C. A., Basavapathruni, A., Jin, L., Boriack-Sjodin, P. A., ... Pollock, R. M. (2013). Potent inhibition of DOT1L as treatment of MLL-fusion leukemia. Blood, 122(6), 1017-1025. doi: 10.1182/blood-2013-04-497644

Damato, B. E., \& Coupland, S. E. (2012). Ocular melanoma. Saudi Journal of Ophthalmology, 26(2), 137-144. doi: 10.1016/j.sjopt.2012.02.004

Eckschlager, T., Plch, J., Stiborova, M., \& Hrabeta, J. (2017). Histone Deacetylase Inhibitors as Anticancer Drugs. International Journal of Molecular Sciences, 18(7), 1414. doi: 10.3390/ijms 18071414

Feng, X., Arang, N., Rigiracciolo, D. C., Lee, J. S., Yeerna, H., Wang, Z., ... Gutkind, J. S. (2019). A Platform of Synthetic Lethal Gene Interaction Networks Reveals that the GNAQ Uveal Melanoma Oncogene Controls the Hippo Pathway through FAK. Cancer Cell, 35(3). 1-16. doi: 10.1016/j.ccell.2019.01.009

Ishiguro, K., Kitajima, H., Niinuma, T., Ishida, T., Maruyama, R., Ikeda, H., ... Suzuki, H. (2018). DOT1L inhibition blocks multiple myeloma cell proliferation by suppressing IRF4-MYC signaling. Haematologica, 104(1), 155-165. doi: 10.3324/haematol.2018.191262 
Li, Y., Jia, R., \& Ge, S. (2017). Role of epigenetics in uveal melanoma. International Journal of Biological Sciences, 13(4), 426-433. doi: 10.7150/ijbs.18331

Meyers, R. M., Bryan, J. G., McFarland, J. M., Weir, B. A., Sizemore, A. E., Xu, H., ... Tsherniak, A. (2017). Computational correction of copy number effect improves specificity of CRISPR-Cas9 essentiality screens in cancer cells. Nature Genetics, 49(12), 1779-1784. doi: 10.1038/ng.3984

Nassa, G., Salvati, A., Tarallo, R., Gigantino, V., Alexandrova, E., Memoli, D., ... Weisz, A. (2019). Inhibition of histone methyltransferase DOT1L silences ER gene and blocks proliferation of antiestrogen-resistant breast cancer cells. Science Advances, 5(2). doi: 10.1126/sciadv.aav5590

O’Neil, N. J., Bailey, M. L., \& Hieter, P. (2017). Synthetic lethality and cancer. Nature Reviews Genetics, 18(10), 613-623. doi: 10.1038/nrg.2017.47

Shi, J., Wang, E., Milazzo, J. P., Wang, Z., Kinney, J. B., \& Vakoc, C. R. (2015). Discovery of cancer drug targets by CRISPR-Cas9 screening of protein domains. Nature Biotechnology, 33(6), 661-667. doi: $10.1038 /$ nbt.3235

Stein, E. M., Garcia-Manero, G., Rizzieri, D. A., Tibes, R., Berdeja, J. G., Savona, M. R., ... Tallman, M. S. (2018). The DOT1L inhibitor pinometostat reduces H3K79 methylation and has modest clinical activity in adult acute leukemia. Blood, 131(24), 2661-2669. doi: 10.1182/blood-2017-12-818948

Steinhardt, A. A., Gayyed, M. F., Klein, A. P., Dong, J., Maitra, A., Pan, D., ... Anders, R. A. (2008). Expression of Yes-associated protein in common solid tumors. Human Pathology, 39(11), 15821589. doi: 10.1016/j.humpath.2008.04.012

Stubbs, M. C., Kim, W., Bariteau, M., Davis, T., Vempati, S., Minehart, J., ... Armstrong, S. A. (2015). Selective inhibition of HDAC1 and HDAC2 as a potential therapeutic option for B-ALL. Clinical Cancer Research, 21(10), 2348-2358. doi: 10.1158/1078-0432.ccr-14-1290

Tsherniak, A., Vazquez, F., Montgomery, P. G., Weir, B. A., Kryukov, G., Cowley, G. S., ... Hahn, W. C. (2017). Defining a Cancer Dependency Map. Cell, 170(3). doi: 10.1016/j.cell.2017.06.010

Van Raamsdonk, C. D., Griewank, K. G., Crosby, M. B., Garrido, M. C., Vemula, S., Wiesner, T., ... Bastian, B. C. (2010). Mutations in GNA11 in uveal melanoma. New England Journal of Medicine, 363(23), 2191-2199. doi: 10.1056/NEJMoa1000584

Wang, T., Birsoy, K., Hughes, N. W., Krupczak, K. M., Post, Y., Wei, J. J., ... Sabatini, D. M. (2015). Identification and characterization of essential genes in the human genome. Science, 350(6264), 1096-1101. doi: 10.1126/science.aac7041

Xu, M. Z., Yao, T. J., Lee, N. P. Y., Ng, I. O. L., Chan, Y. T., Zender, L., ... Luk, J. M. (2009). Yesassociated protein is an independent prognostic marker in hepatocellular carcinoma. Cancer, 115(19), 4576-4585. doi: 10.1002/cncr.24495

Zhao, B., Li, L., \& Guan, K.-L. (2010). Hippo signaling at a glance. Journal of Cell Science, 123(23), 4001-4006. doi: 10.1242/jcs.069070

Zhao, B., Wei, X., Li, W., Udan, R. S., Yang, Q., Kim, J., ... Guan, K. L. (2007). Inactivation of YAP oncoprotein by the Hippo pathway is involved in cell contact inhibition and tissue growth control. Genes and Development, 21(21), 2747-2761. doi: 10.1101/gad.1602907 